\title{
Magnetic Resonance in the Spin Excitation Spectrum of Electron-Doped Cuprate Superconductors
}

\author{
J.-P. Ismer ${ }^{1,2}$, Ilya Eremin ${ }^{1,2}$, Enrico Rossi ${ }^{3}$, Dirk K. Morr ${ }^{3}$ \\ 1 Max-Planck Institut für Physik komplexer Systeme, D-01187 Dresden, Germany \\ 2 Institute für Mathematische und Theoretische Physik, \\ Technische Universität Carolo-Wilhelmina zu Braunschweig, 38106 Braunschweig, Germany \\ 3 Department of Physics, University of Illinois at Chicago, Chicago, IL 60607
}

(Dated: June 30, 2021)

\begin{abstract}
We study the emergence of a magnetic resonance in the superconducting state of the electrondoped cuprate superconductors. We show that the recently observed resonance peak in the electrondoped superconductor $\operatorname{Pr}_{0.88} \mathrm{LaCe}_{0.12} \mathrm{CuO}_{4-\delta}$ is consistent with an overdamped spin exciton located near the particle-hole continuum. We present predictions for the magnetic-field dependence of the resonance mode as well as its temperature evolution in those parts of the phase diagram where $d_{x^{2}-y^{2}}$-wave superconductivity may coexist with an antiferromagnetic spin-density wave.
\end{abstract}

PACS numbers: 74.72.-h, 75.40.Gb, 74.20.Rp, 74.20.Fg

Recently, inelastic neutron scattering (INS) experiments on the electron-doped high-temperature superconductors (HTSC) $\operatorname{Pr}_{0.88} \mathrm{LaCe}_{0.12} \mathrm{CuO}_{4-\delta}\left(\mathrm{PLC}_{0.12} \mathrm{CO}\right)$ [1] observed a resonance peak in the superconducting (SC) state, a phenomenon similar to that observed in the holedoped cuprates [2, 3, 4]. While the resonance frequency in $\mathrm{PLC}_{0.12} \mathrm{CO}, \omega_{\text {res }} \approx 11 \mathrm{meV}$, obeys the same scaling with $T_{c}$ as that in the hole-doped HTSC, there exist two significant differences. First, the resonance is confined to a small momentum region around $\mathbf{Q}=(\pi, \pi)$, where it is almost dispersionless. Second, angle-resolved photoemission (ARPES) experiments on $\mathrm{PLC}_{0.11} \mathrm{CO}[5]$ estimated, based on measurements of the leading edge gap, a maximum SC gap located at the "hot spots" [the Fermi surface (FS) points connected by Q] of $\Delta_{\mathrm{hs}} \approx 5 \mathrm{meV}$. Assuming the same $\mathrm{SC}$ gap in $\mathrm{PLC}_{0.12} \mathrm{CO}$, this would suggest that the resonance is located slightly above the onset of the particle-hole $(p-h)$ continuum given by $2 \Delta_{\mathrm{hs}}$, a result which would challenge the interpretation of the resonance as a spin exciton [4, 6 , , 7, 8,, 9$]$. However, the uncertainties in ARPES and INS experiments are currently such that it is not possible to determine the relative magnitude of $\omega_{\text {res }}$ and $2 \Delta_{\text {hs }}$ and thus ascertain the validity of the spin exciton scenario.

In this Letter we address this issue and study the emergence of a resonance mode in the SC state of electrondoped HTSC. We show that the experimental features of the resonance in $\mathrm{PLC}_{0.12} \mathrm{CO}$ can be explained within a spin-exciton scenario. In particular, we demonstrate that the position of the hot spots close to the Brillouin zone (BZ) diagonal 10, 11] combined with the momentum dependence of the fermionic interaction leads to an almost dispersionless resonance that is confined to a small momentum region around $\mathbf{Q}$. Moreover, while the resonance is always located below the $p$ - $h$ continuum in systems with a quasiparticle lifetime, $1 / \Gamma \rightarrow \infty$, we show that the maximum of the resonance's intensity can be shifted to frequencies above the $p$ - $h$ continuum when $1 / \Gamma$ is suf- ficiently small. In this case, the form of the spin susceptibility is more reminiscent of the magnetic coherence effect in $\mathrm{La}_{2-x} \mathrm{Sr}_{x} \mathrm{CuO}_{4}$ [12, 13] than of the resonance observed in the hole-doped HTSC. We present two predictions for further experimental tests of the spin exciton scenario. First, we show that a magnetic field in the $a b$ plane leads to an energy splitting of the resonance which for typical fields is sufficiently large to be experimentally observable in the electron-doped HTSC. Second, we predict that in those parts of the phase diagram, where $d_{x^{2}-y^{2}}$-wave superconductivity $(d \mathrm{SC})$ coexists with an antiferromagnetic spin-density wave (SDW) [14, 15, 16] and $T_{N}<T_{c}$, the resonance evolves into the Goldstone mode of the SDW state as $T_{N}$ is approached.

The starting point for our study of the resonance mode in the electron-doped cuprates is the Hamiltonian

$$
H=\sum_{\mathbf{k} \sigma} \varepsilon_{\mathbf{k}} c_{\mathbf{k}, \sigma}^{\dagger} c_{\mathbf{k}, \sigma}+\sum_{\mathbf{k}} \Delta_{\mathbf{k}} c_{\mathbf{k}, \uparrow}^{\dagger} c_{-\mathbf{k}, \downarrow}^{\dagger}+\text { H.c. },
$$

where $c_{\mathbf{k}, \sigma}^{\dagger}$ creates an electron with spin $\sigma$ and momentum $\mathbf{k}$, and $\Delta_{\mathbf{k}}$ is the SC gap with $d_{x^{2}-y^{2}}$-wave symmetry. The normal state tight binding dispersion

$$
\begin{aligned}
\epsilon_{\mathbf{k}}= & -2 t\left(\cos k_{x}+\cos k_{y}\right)-4 t^{\prime} \cos k_{x} \cos k_{y} \\
& -2 t^{\prime \prime}\left(\cos 2 k_{x}+\cos 2 k_{y}\right)-\mu
\end{aligned}
$$

with $t=250 \mathrm{meV}, t^{\prime} / t=-0.4, t^{\prime \prime} / t=0.1$ and $\mu / t=$ -0.2 reproduces the position of the hot spots and the underlying FS [Fig. 1(a)] as inferred from ARPES [5].

Despite the same FS topology in the electron-doped and hole-doped cuprates, the angular dependence of the superconducting gap along the FS is qualitatively different in these systems. Based on a scenario in which superconductivity arises from the exchange of antiferromagnetic spin fluctuations, it was argued that the maximum SC gap is achieved near the hot spots [11, 17, 18]. In the hole-doped cuprates, the hot spots are located close 
to $\mathbf{q}=( \pm \pi, 0)$ and $(0, \pm \pi)$, resulting in a SC $d_{x^{2}-y^{2} \text {-wave }}$ gap that varies monotonically along the FS, as shown in Fig. 1(b). In contrast, in the electron-doped cuprates, the hot spots are located much closer to the zone diagonal [Fig. 1(a)], leading to a nonmonotonic behavior of the SC gap [11, 18], in agreement with ARPES experiments [5]. A good fit of $\Delta_{\mathbf{k}}$ to the experimental data is achieved via the inclusion of a higher harmonic, such that $\Delta_{\mathbf{k}}=\frac{\Delta_{0}}{2}\left(\cos k_{x}-\cos k_{y}\right)+\frac{\Delta_{1}}{2}\left(\cos 2 k_{x}-\cos 2 k_{y}\right)$, where $\Delta_{1} / \Delta_{0}=0.63$ ensures that the maximum of $\left|\Delta_{\mathbf{k}}\right|$ along the FS is located at the hot spots, as shown in Fig. 1)(b) [19]. Since the magnitude of the $\mathrm{SC}$ gap in $\mathrm{PLC}_{0.12} \mathrm{CO}$ is still unknown, we use $\Delta_{0}=10 \mathrm{meV}$ which yields $\Delta_{\mathrm{hs}}=5$ meV thus reproducing the ARPES estimate of the SC gap at the hot spots of $\mathrm{PLC}_{0.11} \mathrm{CO}$. Note that the results shown below are robust against (reasonable) changes in the band structure, as long as $\Delta_{\mathrm{hs}}$ remains unchanged.
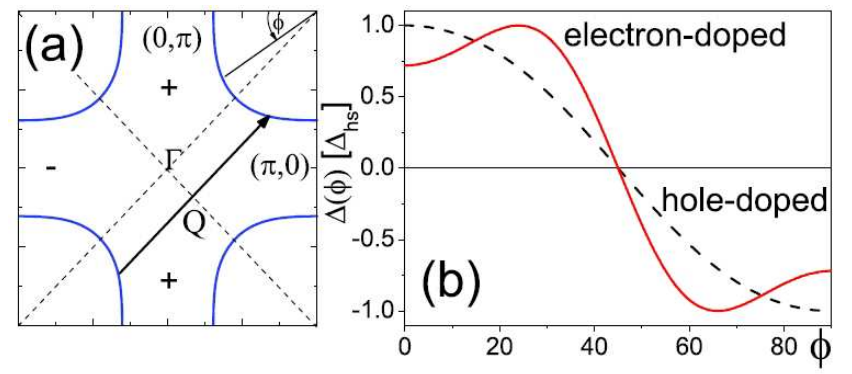

FIG. 1: (color online). (a) Fermi surface in optimally electrondoped cuprates. The arrow indicates the scattering of quasiparticles by Q. (b) Angular dependence of the SC gap for electron and hole-doped HTSC.

Similar to the hole-doped HTSC [4, 6, 7, 8] the resonance peak in the SC state of the electron-doped cuprates can be understood by considering the dynamical spin susceptibility within the random phase approximation (RPA)

$$
\chi(\mathbf{q}, \omega)=\frac{\chi_{0}(\mathbf{q}, \omega)}{1-U(\mathbf{q}) \chi_{0}(\mathbf{q}, \omega)},
$$

where $U(\mathbf{q})$ is the fermionic four-point vertex and $\chi_{0}(\mathbf{q}, \omega)$ is the free-fermion susceptibility given by the sum of two single bubble diagrams consisting of either normal or anomalous Green functions. While momentum independent as well as momentum dependent forms of $U(\mathbf{q})$ were used in the hole-doped cuprates [4, 6,, 7, , 8 ], the close proximity of the (commensurate) antiferromagnetic and SC phases in the electron-doped HTSC suggests that $U(\mathbf{q})$ is momentum dependent, with a maximum at $\mathbf{Q}=(\pi, \pi)[20]$. Here, we use $U_{\mathbf{q}}=-\frac{U_{0}}{2}\left(\cos q_{x}+\cos q_{y}\right)$ which reproduces a nearly dispersionless resonance mode around Q. The form of $\chi_{0}$ in the hole-doped and electron-doped HTSC is qualitatively similar, and has been extensively discussed for the former [4, 6, 7, , 8]. For momenta $\mathbf{q}$ near $\mathbf{Q}$ and $\Gamma=0^{+}, \operatorname{Im} \chi_{0}$ is zero at low fre- quencies and exhibits a discontinuous jump at the onset frequency of the $p$ - $h$ continuum $\Omega_{c}(\mathbf{q})=\left|\Delta_{\mathbf{k}}\right|+\left|\Delta_{\mathbf{k}+\mathbf{q}}\right|$, where both $\mathbf{k}$ and $\mathbf{k}+\mathbf{q}$ lie on the FS [for $\mathbf{q}=\mathbf{Q}$ one has $\left.\Omega_{c}(\mathbf{Q})=2 \Delta_{\mathrm{hs}}\right]$. The discontinuity in $\operatorname{Im} \chi_{0}$, which is a direct consequence of $\operatorname{sgn}\left(\Delta_{\mathbf{k}}\right)=-\operatorname{sgn}\left(\Delta_{\mathbf{k}+\mathbf{q}}\right)$ and hence the $d_{x^{2}-y^{2}}$-wave symmetry of the SC gap, leads to a logarithmic singularity in $\operatorname{Re} \chi_{0}$. As a result, the resonance conditions (i) $U_{\mathbf{Q}} \operatorname{Re} \chi_{0}\left(\mathbf{Q}, \omega_{\text {res }}\right)=1$ and (ii) $\operatorname{Im} \chi_{0}\left(\mathbf{Q}, \omega_{\text {res }}\right)=0$ can be fulfilled simultaneously at $\omega_{\text {res }}<\Omega_{c}$ for any $U_{\mathbf{Q}}>0$, leading to the emergence of a resonance peak as a spin exciton. Note that for finite $\Gamma$, condition (i) can only be satisfied if $U_{\mathbf{Q}}$ exceeds a critical value, and condition (ii) is replaced by $U_{\mathbf{Q}} \operatorname{Im} \chi_{0}\left(\mathbf{Q}, \omega_{\text {res }}\right) \ll 1$.
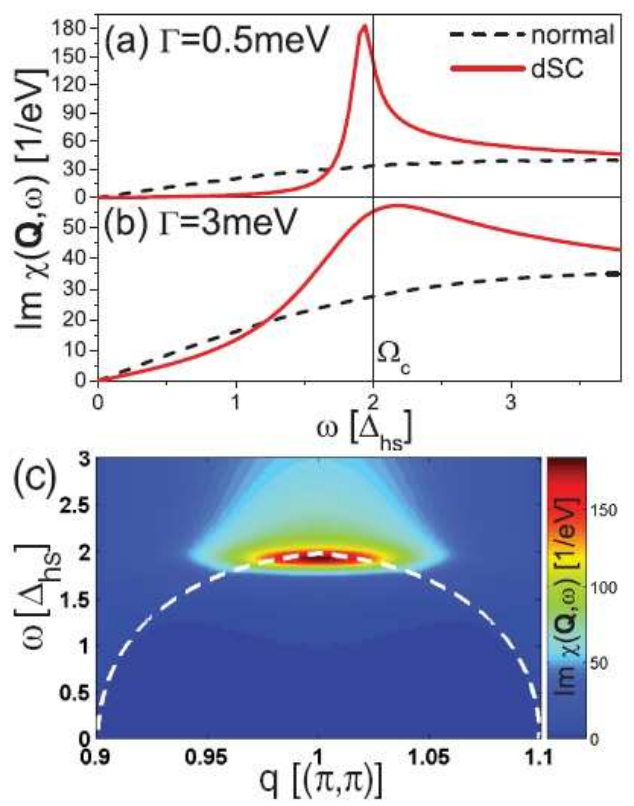

FIG. 2: (color online). $\operatorname{Im} \chi(\mathbf{Q}, \omega)$ for $U_{0}=0.854 \mathrm{eV}$ and (a) $\Gamma=0.5 \mathrm{meV}$, and (b) $\Gamma=3 \mathrm{meV}$. (c) Contour plot of $\operatorname{Im} \chi$ in the $(\omega, \mathbf{q})$ plane for the parameters in (a), together with $\Omega_{c}(\mathbf{q})$ (dashed white line).

In Fig. 2, we present $\operatorname{Im} \chi(\mathbf{Q}, \omega)$ resulting from Eq. (3). Since the INS data suggest that the resonance is located close to the $p$ - $h$ continuum, we have chosen $U_{0}=0.854$ $\mathrm{eV}$ such that for $\Gamma \rightarrow 0$ (not shown), the resonance is located at $\omega_{\text {res }}=9.8 \mathrm{meV}=0.98 \Omega_{c}$. For small $\Gamma=0.5$ meV [Fig. 2(a)], the resonance broadens but only exhibits a negligible frequency shift. However, for larger $\Gamma=3 \mathrm{meV}$ [Fig. 2(b)], the resonance has not only become much broader but its peak intensity has also shifted to $\omega_{\text {res }} \approx 11 \mathrm{meV}=1.1 \Omega_{c}$ well above the onset of the $p$ - $h$ continuum. In this case, neither of the resonance conditions is satisfied, and $\operatorname{Im} \chi$ is more reminiscent of the magnetic coherence effect in $\mathrm{La}_{2-x} \mathrm{Sr}_{x} \mathrm{CuO}_{4}$ [12, 13] than of the resonance in the hole-doped HTSC. Hence, when the resonance is located close to the $p$ - $h$ continuum, its form depends rather sensitively on $\Gamma$. 
The observed spectral weight in $\operatorname{Im} \chi$ at frequencies much below $\Omega_{c}$ [1] is consistent with a shorter lifetime $1 / \Gamma$, arising, for example, from disorder effects. Whether the INS data are better described by the results shown in Fig. 2(a) (albeit with a larger $\Delta_{\text {hs }}$ such that the experimentally determined $\omega_{\text {res }}=11 \mathrm{meV}$ corresponds to $0.98 \Omega_{c}$ ), or in Fig. 2(b), is presently unclear, mainly due to experimental resolution effects which are in general difficult to account for. Moreover, since the resonance's maximum intensity is affected by its distance to the $p$ - $h$ continuum, the band structure, the magnitude of the SC gap, and $1 / \Gamma$, we expect it to be smaller in the electrondoped HTSC than in the hole-doped cuprates.

In Fig. 2(c) we present a contour plot of $\operatorname{Im} \chi$ for $\Gamma=$ $0.5 \mathrm{meV}$ along $\mathbf{q}=\eta(\pi, \pi)$ together with the momentum dependence of $\Omega_{c}(\mathbf{q})$, the onset of the $p-h$ continuum. The resonance is almost dispersionless and exists only in a small momentum region $(0.96 \mathbf{Q} \lesssim \mathbf{q} \lesssim 1.04 \mathbf{Q})$ around $\mathbf{Q}$, in agreement with experiment [1]. This effect arises from the momentum dependence of $U(\mathbf{q})$, combined with the fact that the resonance at $\mathbf{Q}$ is located only slightly below the $p$ - $h$ continuum, which leads to a "merging" of the resonance with the $p$ - $h$ continuum at small deviations from $\mathbf{Q}$. The momentum connecting the nodal points, $\mathbf{q}_{n} \approx 0.9 \mathbf{Q}$, where $\Omega_{c}(\mathbf{q})$ reaches zero, is much closer to $\mathbf{Q}$ than in the hole-doped systems where $\mathbf{q}_{n} \approx 0.8 \mathbf{Q}$, leading to an additional narrowing of the dispersion.

The phase diagram of the electron-doped HTSC, and a SC gap that is much smaller than in the holed-doped cuprates, provide further opportunities for testing the nature of the resonance peak. Consider, for example, the effects of a magnetic field $H$ in the $a b$ plane, which enters the calculation of $\chi$ only through the Zeeman splitting of the electronic bands, while orbital effects are absent 21]. The field lifts the degeneracy of the transverse and longitudinal components of $\chi_{0}$ which are given by

$$
\begin{aligned}
\chi_{0}^{ \pm \mp}\left(\mathbf{q}, i \omega_{n}\right)= & -\frac{1}{N} \sum_{\mathbf{k}}\left\{c^{+} \frac{f_{\mathbf{k}+\mathbf{q}}^{ \pm}-f_{\mathbf{k}}^{\mp}}{i \omega_{n}+\xi_{\mathbf{k}+\mathbf{q}}^{ \pm}-\xi_{\mathbf{k}}^{\mp}}\right. \\
& +\frac{c^{-}}{2} \frac{1-f_{\mathbf{k}+\mathbf{q}}^{\mp}-f_{\mathbf{k}}^{\mp}}{i \omega_{n}-\xi_{\mathbf{k}+\mathbf{q}}^{\mp}-\xi_{\mathbf{k}}^{\mp}} \\
& \left.-\frac{c^{-}}{2} \frac{1-f_{\mathbf{k}+\mathbf{q}}^{ \pm}-f_{\mathbf{k}}^{ \pm}}{i \omega_{n}+\xi_{\mathbf{k}+\mathbf{q}}^{ \pm}+\xi_{\mathbf{k}}^{ \pm}}\right\}, \\
\chi_{0}^{z z}\left(\mathbf{q}, i \omega_{n}\right)= & -\frac{1}{4 N} \sum_{\mathbf{k}}\left\{c^{+} \frac{f_{\mathbf{k}+\mathbf{q}}^{+}-f_{\mathbf{k}}^{+}}{i \omega_{n}+E_{\mathbf{k}+\mathbf{q}}-E_{\mathbf{k}}}\right. \\
& +c^{+} \frac{f_{\mathbf{k}+\mathbf{q}}^{-}-f_{\mathbf{k}}^{-}}{i \omega_{n}+E_{\mathbf{k}+\mathbf{q}}-E_{\mathbf{k}}} \\
& +c^{-} \frac{1-f_{\mathbf{k}+\mathbf{q}}^{-}-f_{\mathbf{k}}^{+}}{i \omega_{n}-E_{\mathbf{k}+\mathbf{q}}-E_{\mathbf{k}}} \\
& \left.-c^{-} \frac{1-f_{\mathbf{k}+\mathbf{q}}^{+}-f_{\mathbf{k}}^{-}}{i \omega_{n}+E_{\mathbf{k}+\mathbf{q}}+E_{\mathbf{k}}}\right\}
\end{aligned}
$$

$$
\text { commensurate SDW }[14,15,16] \text {. Extending the spin- }
$$
exciton scenario to such a coexistence phase [26], we find that the spin response at $(\pi, \pi)$ only possesses a Goldstone mode at zero energy but no additional resonance at higher energies. Since the existence of a Goldstone mode requires the condition $U_{\mathbf{Q}} \operatorname{Re} \chi_{0}(\mathbf{Q}, \omega)=1$ to be satisfied at $\omega=0$, and since $\operatorname{Re} \chi_{0}(\mathbf{Q}, \omega)$ increases monotonically with frequency from $\omega=0$ up to $\Omega_{c}$, the resonance conditions can only be satisfied once, namely at $\omega_{\text {res }}=0$. In contrast, in a "pure" SC state, the reso- 
nance is necessarily located at $\omega_{\text {res }} \neq 0$. Moreover, the results of recent experiments [16, 25] suggest the existence of electron-doped HTSC with $T_{N}<T_{c}$. For these materials, it follows from the above discussion that the resonance of the pure SC state shifts downward in energy with decreasing temperature, until it reaches zero energy at $T_{N}$ and forms the Goldstone mode. Within the spin exciton scenario, this requires that $U(\mathbf{q})$ increases with decreasing temperature. In order to exemplify the resonance's temperature evolution, we consider a system with $T_{N}<T_{c}$ (for concreteness we chose $T_{N}=T_{c} / 6$ ). We assume (somewhat arbitrarily) that $U_{0}$ varies linearly with temperature while the momentum dependence of $U(\mathbf{q})$ remains unchanged, such that the resonance at $\mathbf{Q}$ and $T=0.75 T_{c}$ is located at $\omega_{\text {res }} \approx 0.92 \Omega_{c}$, while for $T=T_{N}$, we have $\omega_{\text {res }}=0$. In Fig. 4, we present the contour plots of $\operatorname{Im} \chi$ for four different temperatures. While the res-

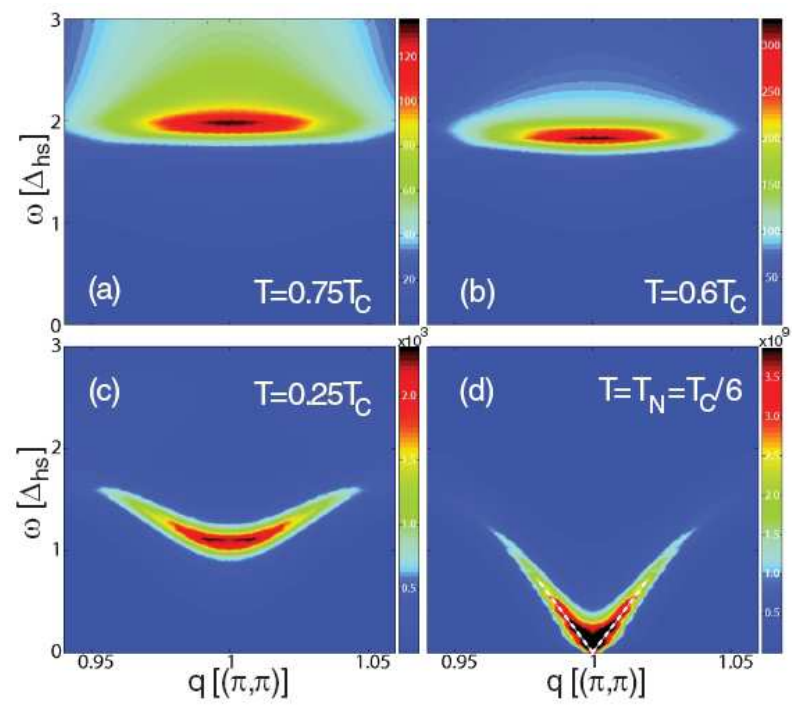

FIG. 4: (color online). Contour plots of $\operatorname{Im} \chi$ (in units of $1 / \mathrm{eV}$ ) for $\Gamma=0.5 \mathrm{meV}$ at four different temperatures and $U_{0}(T)=0.8784 \mathrm{eV}-0.0014 \mathrm{eV} / \mathrm{K} \cdot \mathrm{T}$. The white dashed line in (d) represents the dispersion of the Goldstone mode.

onance shifts downwards with decreasing temperature, it remains nearly dispersionless over some temperature range below $T_{c}$ [see Figs. 4(a) and 4(b)]. However, upon decreasing temperature even further [Fig. 4(c)], the "flat" dispersion evolves continuously into an upward dispersion whose minimum is located at $\mathbf{Q}$. Finally, at $T_{N}$ [Fig. 4(d)], the minimum of the dispersion at $\mathbf{Q}$ reaches zero energy and the resonance becomes the Goldstone mode of the SDW state [27]. The qualitative features of this evolution are independent of the specific temperature dependence of $U_{0}$, as long as $U(\mathbf{q})$ decreases sufficiently fast with deviation from $(\pi, \pi)$.

In summary, we studied the emergence of a magnetic resonance mode in the $\mathrm{SC}$ state of the electron-doped HTSC. We show that the recently observed resonance peak in $\mathrm{PLC}_{0.12} \mathrm{CO}$ is likely an overdamped spin exciton located near the $p$ - $h$ continuum. We discuss the magnetic-field dependence of the resonance as well as its temperature evolution in those parts of the phase diagram where $d \mathrm{SC}$ may coexist with a antiferromagnetic SDW.

We would like to thank A. Chubukov, F. Krüger, P. Dai and J. Zaanen for helpful discussions and P. Dai and R. L. Greene for sharing their experimental data prior to publication. This work was supported by the DAAD under Grant No. D/05/50420, the Alexander von Humboldt Foundation, the NSF under Grant No. DMR0513415, the U.S. DOE under Award No. DE-FG0205ER46225, and the IMPRS on "Dynamic Processes in Atoms, Molecules, and Solids".

[1] S.D. Wilson et al., Nature (London) 442, 59 (2006).

[2] J. Rossat-Mignod et al., Physica (Amsterdam) 185189C, 86 (1991).

[3] H.A. Mook, et al., Phys. Rev. Lett. 70, 3490 (1993); P. Bourges et al., Phys. Rev. B 53, 876 (1996); P. Dai et al., Phys. Rev. Lett. 77, 5425 (1996); H.F. Fong et al., Nature (London) 398, 588 (1999); P. Dai et al., Science 284, 1344 (1999); H. He et al., Science 295, 1045 (2002); S. Pailhès et al., Phys. Rev. Lett. 93, 167001 (2004); S.M. Hayden et al., Nature (London) 429, 531 (2004); C. Stock et al., Phys. Rev. B 69, 014502 (2004).

[4] H.F. Fong et al., Phys. Rev. Lett. 75, 316 (1995).

[5] H. Matsui et al., Phys. Rev. Lett. 95, 017003 (2005).

[6] D.Z. Liu, Y. Zha, and K. Levin, Phys. Rev. Lett. 75, 4130 (1995); A.J. Millis and H. Monien, Phys. Rev. B 54, 16172 (1996); A. Abanov and A.V. Chubukov, Phys. Rev. Lett. 83, 1652 (1999); J. Brinckmann and P.A. Lee, Phys. Rev. Lett. 82, 2915 (1999); I. Sega, P. Prelovšek, and J. Bonca, Phys. Rev. B 68, 054524 (2003).

[7] M.R. Norman, Phys. Rev. B 61, 14751 (2000); 63, 092509 (2001)

[8] I.Eremin et al., Phys. Rev. Lett. 94, 147001 (2005).

[9] J.-X. Li, J. Zhang, and J. Luo, Phys. Rev. B 68, 224503 (2003).

[10] F. Onufrieva and P. Pfeuty, Phys. Rev. Lett. 92, 247003 (2004).

[11] P. Krotkov and A.V. Chubukov, Phys. Rev. Lett. 96, 107002 (2006); Phys. Rev. B 74, 014509 (2006).

[12] T.E. Mason et al., Phys. Rev. Lett. 77, 1604 (1996); B. Lake et al., Nature (London) 400, 43 (1999).

[13] D.K. Morr and D. Pines, Phys. Rev. B 61, R6483 (2000); 62, 15177 (2000).

[14] G.M. Luke et al., Phys. Rev. B 42, 7981 (1990).

[15] P.K. Mang et al., Phys. Rev. Lett. 93, 027002 (2004).

[16] S.D. Wilson et al., Phys. Rev. B 74, 144514 (2006).

[17] G. Blumberg et al., Phys. Rev. Lett. 88, 107002 (2002).

[18] D. Manske, I. Eremin, and K.-H. Bennemann, Phys. Rev. B 62, 13922 (2000).

[19] Similar results are also obtained for other higher harmonic terms in $\Delta_{\mathbf{k}}$, see also A.P. Schnyder et al., Phys. Rev. B 70, 214511 (2004).

[20] E.M. Motoyama et al., Nature (London) 445, 186 (2007). 
[21] For orbital effects, see M. Eschrig, M.R. Norman, and B. Janko, Phys. Rev. B 64, 134509 (2001).

[22] The effect of $H$ on the Fermi-functions is negligible around the hot spots, at least for $T \ll T_{c}$.

[23] Note that in H.-M. Jiang and J.-X. Li, Phys. Rev. B 73, 224507 (2006) no splitting of the resonance was found since only $\chi^{ \pm}$was considered.

[24] P. Li, F. Balakirev, and R.L. Greene, Phys. Rev. B 75,
172508 (2007).

[25] P. Dai (private communication).

[26] J.R. Schrieffer, X.G. Wen, and S.C. Zhang, Phys. Rev. B 39, 11663 (1989).

[27] Note that for a momentum independent $U(\mathbf{q})$, the emerging SDW state is incommensurate. 